\title{
Aplicación práctica de bpm para la mejora del subproceso de picking en un centro de distribución logístico
}

\begin{abstract}
RESUMEN
Los centros de distribución son áreas muy sensibles a los errores cometidos por operarios en la preparación de pedidos, teniendo un fuerte impacto en el nivel de servicio y los objetivos de la organización. En el caso de una botica es relevante debido a la gran cantidad de productos con los que trabaja y a la alta demanda con que se presentan los pedidos. Como herramienta de solución a esta problemática se plantea la aplicación de la Gestión de Procesos de Negocio (BPM - Business Process Management), obteniendo como resultados la caracterización de procesos de la organización, el diagrama actual del subproceso de picking (ASIS) y el diagrama con la mejora de añadir un punto de control adicional (TO-BE) aplicando la notación BPMN (Business Process Management Notation).

Palabras clave: BPM, BPMN, Picking, AS-IS, TO-BE
\end{abstract}

PRACTICAL APPLICATION OF BPM TO IMPROVE PICKING PROCESS IN A LOGISTICS DISTRIBUTION CENTER

\section{ABSTRACT}

Distribution centers are quite sensitive areas to produce failures due to human mistakes through the picking process. As a result, it impacts enormously the level of service and organization's goals. The object of study is a drugstore whose number of orders is very high and the number of handled products too. Solution is given as a tool that applies Business Process Management (BPM) and provides characterization of organizational processes, the current picking process diagram (AS-IS) and the improved diagram (TO-BE) which enhances the process adding a new control point. Besides, in this paper BPMN notation was applied correctly in each diagram.

Keywords: BPM, BPMN, Picking, AS-IS, TO-BE

\section{INTRODUCCIÓN}

Business Process Management o BPM (Gestión de Procesos de Negocios) es una disciplina y conjunto de buenas prácticas, metodologías y tecnologías que permiten una adecuada gestión de los procesos empresariales. La aplicación de BPM logra obtener una organización cuyo enfoque pasa de una tradicional estructura jerárquica y funcional a una más eficiente, eficaz, articulada y comprometida con el cliente. Es decir, una gestión por procesos.

Como aplicación práctica se escogió el centro de distribución de una conocida cadena de boticas del medio local. Para ello, se realizaron entrevistas, se levantó información relevante y se procedió a la identificación de los macro procesos de la organización. El subproceso de picking es sumamente importante ya que es la razón de ser del negocio. Sin embargo, se presentan errores en esta etapa.

La solución se inicia en la caracterización del subproceso de picking, luego se elabora el diagrama AS-IS (versión actual, en el idioma inglés significa "como es"), finalmente el diagrama TO$\mathrm{BE}$ (versión con mejora, en el idioma inglés significa "como va a ser"), que es el objetivo de la solución a las fallas presentadas en la preparación de los pedidos del centro de distribución, logrando aplicar correctamente la notación BPMN.

\section{DEFINICIONES}

\subsection{GESTIÓN DE PROCESOS}

La gestión de procesos se define como un sistema completo cuyo principal objetivo es enfocarse en la mejora continua del funcionamiento de las actividades de una organización. Como resultado se obtiene una reducción de la variabilidad innecesaria en los productos finales, así como el uso óptimo de recursos y eliminación de actividades repetitivas.

\subsection{ENFOQUE DE PROCESOS}

El enfoque por procesos busca que la organización completa se visualice desde el punto de vista del cliente. En el típico enfoque funcional cada área es considerada independiente, responsable únicamente de sus procesos, y no hay una adecuada interconexión y articulación con las demás áreas. Sin embargo, el enfoque por procesos rompe esas barreras entre áreas mejorando y

\footnotetext{
Ingeniero Informático, PUCP. Analista Programador, SUNAT. E-mail: fsantos@pucp.edu.pe
} 2 Docente Principal, Facultad de Ingeniería Industrial, UNMSM. E-mail: esantosd@unmsm.edu.pe 
rediseñando los procesos con el fin de lograr mejorar actividades muy arraigadas de las áreas funcionales; generar una estructura organizacional más dinámica, flexible; generar mayor valor y lograr la satisfacción del cliente. La figura 1 muestra lo que se pretende con este enfoque.

Figura 1. Enfoque Funcional vs Enfoque de Procesos

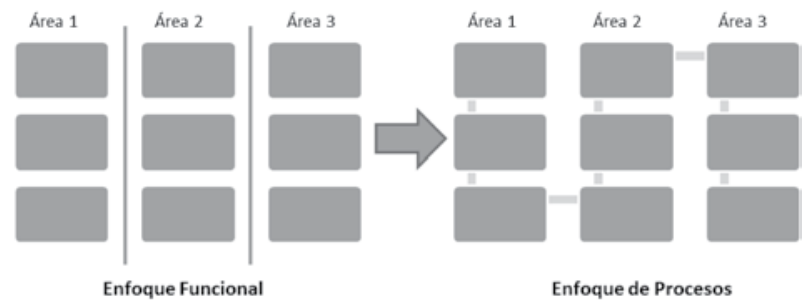

Fuente: Elaboración propia

\subsection{BPM}

Gartner define BPM como "una disciplina de gestión que trata los procesos de negocio como activos que mejoran directamente el desempeño de la organización por el manejo operativo de excelencia y la agilidad del negocio" [2] (traducido del idioma inglés).

Al igual que el Ciclo de Deming (Planificar, Hacer, Verificar y Actuar) aplicado a un sistema de gestión de calidad, BPM posee su propio ciclo denominado "Ciclo de Vida de la Administración de los Procesos de Negocio" como se muestra en la figura 2.

Figura 2. Ciclo BPM

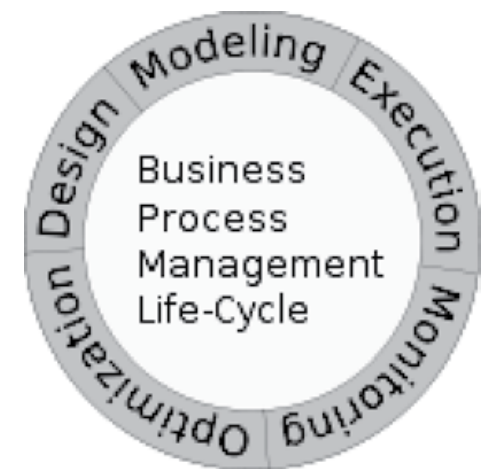

Fuente: Tomado de Rabu T. [5]

Figura 3: Ciclo de Deming

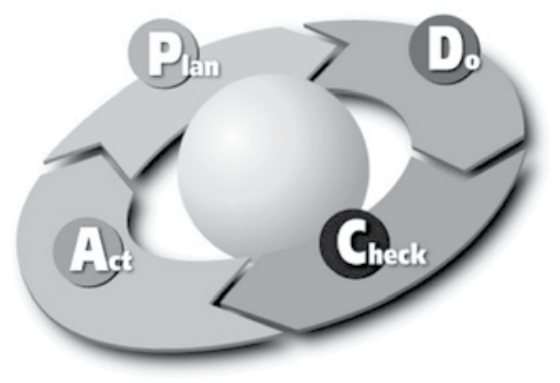

Fuente: Tomado de Bulsuk K. [1]
Las etapas del ciclo BPM son las siguientes [5] (traducido del idioma inglés):

- Diseñar: Esta etapa contempla la identificación existente y diseño de los diagramas del proceso. Incluye la representación del flujo, los actores involucrados, alertas, notificaciones, procedimientos estandarizados y las tareas (manuales y automatizadas).

- Modelar: Modelamiento toma el diseño de la etapa previa e introduce una combinación de variables. Por ejemplo, cambios en los costos de los materiales con la finalidad de apreciar como el proceso se desempeña ante nuevos escenarios.

- Ejecutar: Mediante un sistema informático se automatiza la ejecución de los pasos del proceso, se envía las tareas a cada responsable controlando su tiempo de ejecución y el proceso en general. Se debe tener en cuenta las reglas del negocio pre establecidas.

- Monitorear: Abarca el seguimiento de los procesos individuales, mediante información accesible y estadísticas con el fin de relacionarlos con las estrategias de la organización.

- Optimizar: Incluye la lectura de la información correspondiente al rendimiento del proceso, identificando los actuales y potenciales cuellos de botella, así mismo, las posibles soluciones en reducción de costos u otras mejoras con la finalidad de aplicar soluciones en el diseño del proceso.

Es importante recalcar la fuerte relación de los ciclos presentados en las figuras 2 y 3 , debido a que dentro del ciclo BPM se aplica constantemente la mejora continua desarrollada por Deming.

El símil entre el ciclo BPM y el ciclo de Deming esta dado en relacionar directamente sus etapas, tal como se aprecia en la tabla 1:

Tabla 1. Comparación de los ciclos BPM y Deming

\begin{tabular}{|c|c|}
\hline Etapa BPM & Etapa Deming \\
\hline Diseño y Modelamiento & Planificar \\
\hline Ejecución & Hacer \\
\hline Monitoreo & Verificar \\
\hline Optimización & Actuar \\
\hline
\end{tabular}

Fuente: Elaboración propia 


\subsection{BPMN}

Business Process Management Notation es conocida como la notación de BPM. "Es la notación estándar (grupo de iconos y gráficos) para modelar los procesos de negocio. El principal objetivo de este estándar es su uso para el modelamiento común de gráficos a lo largo de todo el modelamiento del proceso de negocio mediante herramientas y aplicaciones BPM, por ello BPMN es complementario a otros estándares BPM" [3]. BPMN se divide en cuatro categorías que se muestran en la tabla 2:

Tabla 2. Categorías de BPMN

\begin{tabular}{|c|c|c|c|}
\hline Categoría & Descripción & Elemento & Notación BPM \\
\hline \multirow{3}{*}{ Objetos de Flujo } & \multirow{3}{*}{$\begin{array}{l}\text { Elementos que definen el } \\
\text { comportamiento de los procesos. }\end{array}$} & Actividad & \\
\hline & & Evento & \\
\hline & & Compuerta & \\
\hline \multirow{3}{*}{ Objetos de Conexión } & \multirow{3}{*}{$\begin{array}{l}\text { Elementos que permiten conectar } \\
\text { los objetos de flujo que se dan en } \\
\text { el desarrollo de la estructura del } \\
\text { proceso. }\end{array}$} & Flujo de secuencia & \\
\hline & & Flujo de mensaje & \\
\hline & & Asociación & \\
\hline \multirow{2}{*}{ Carriles } & \multirow{2}{*}{$\begin{array}{l}\text { Elementos que permiten organizar } \\
\text { las actividades separadamente para } \\
\text { apreciar más claramente los procesos } \\
\text { y roles. }\end{array}$} & Pool & $\bar{g}$ \\
\hline & & Lame & $\bar{\vdots}$ \\
\hline \multirow{3}{*}{ Artefactos } & \multirow{3}{*}{$\begin{array}{l}\text { Elementos que permiten ofrecer } \\
\text { información adicional de los procesos, } \\
\text { dando mayor claridad. }\end{array}$} & Objeto de datos & \\
\hline & & Grupo & \\
\hline & & Anotación & \\
\hline
\end{tabular}

Fuente: Tomado del Object Management Group [4]

De las cuatro categorías señaladas en la tabla 2, la categoría de "Objetos de Flujo" posee una serie de variantes y aplicaciones particulares para cada uno de sus elementos. A continuación se muestra en la tabla 3 las variantes más usadas:

\section{APLICACIÓN DE BPM}

\subsection{MAPEO DE MACROPROCESOS}

Se define el mapa de procesos como una gráfica que representa las actividades que hacen funcionar a la organización, refiriéndose tanto a las actividades internas como externas. El correcto diseño del diagrama está asociado al adecuado alineamiento a la visión y planificación estratégica de la organización.

El mapeo de macro procesos contiene tres tipos de procesos:
- Procesos estratégicos: Se refiere a todos aquellos que involucran políticas, tomas de decisiones, planificación y alta gestión.

- Procesos clave u operativos: Son los procesos del quehacer diario, operacionales y que generan valor.

- Procesos de apoyo: Son los procesos que proporcionan el soporte y recursos a los procesos claves u operativos.

El centro de distribución seleccionado se identifica mediante el mapa de procesos ilustrado en la figura 4. 
Tabla 3. Variantes de los objetos de flujo

\begin{tabular}{|c|c|c|c|}
\hline Elemento & Variante & Descripción & Notación BPM \\
\hline \multirow{5}{*}{ Actividad } & Actividad de servicio & $\begin{array}{l}\text { Enlaza a algún tipo de servicio, el cual puede ser un Web } \\
\text { Service o una aplicación automatizada. }\end{array}$ & \\
\hline & Actividad de usuario & $\begin{array}{l}\text { Una típica actividad de "flujo de trabajo" donde un humano } \\
\text { lleva a cabo la tarea por medio de alguna tecnología. }\end{array}$ & (3) \\
\hline & Actividad manual & $\begin{array}{l}\text { Una actividad no automatizada que un humano lleva a } \\
\text { cabo. }\end{array}$ & 妇 \\
\hline & Subproceso colapsado & $\begin{array}{l}\text { Tiene la forma de una actividad con una cruz pequeña en la } \\
\text { parte central baja. El detalle de este subproceso es visible } \\
\text { en otro diagrama. }\end{array}$ & + \\
\hline & $\begin{array}{l}\text { Actividad de múltiples } \\
\text { instancias }\end{array}$ & $\begin{array}{l}\text { Es una actividad que repite en paralelo una cantidad } \\
\text { determinada y conocida de veces. }\end{array}$ & III \\
\hline \multirow{4}{*}{ Evento } & Evento de inicio & $\begin{array}{l}\text { El evento de inicio muestra cuando un proceso puede } \\
\text { ocurrir. Se diagrama como un círculo abierto de una } \\
\text { delgada y simple línea. }\end{array}$ & \\
\hline & Evento de fin & $\begin{array}{l}\text { Un evento de fin marca donde un proceso concluye. Se } \\
\text { representa mediante un círculo abierto de una línea simple } \\
\text { y un poco más gruesa que el de Evento de inicio. }\end{array}$ & \\
\hline & Evento fin por compensación & $\begin{array}{l}\text { Define dos mensajes: El fin del proceso y el motivo, que en } \\
\text { este caso viene a ser por un error suscitado y que requiere } \\
\text { ser corregido o compensado. }\end{array}$ & \\
\hline & $\begin{array}{l}\text { Evento intermedio por } \\
\text { compensación }\end{array}$ & $\begin{array}{l}\text { Define dos mensajes: La interrupción del proceso en una } \\
\text { actividad intermedia y su compensación. }\end{array}$ & \\
\hline \multirow{2}{*}{ Compuerta } & Compuerta exclusiva & $\begin{array}{l}\text { Es una compuerta que se utiliza como divergencia, es decir } \\
\text { solo activa un camino. }\end{array}$ & \\
\hline & Compuerta paralela & $\begin{array}{l}\text { Compuerta que indica que varias actividades pueden } \\
\text { realizarse paralelamente. }\end{array}$ & \\
\hline
\end{tabular}

Fuente: Tomado de White S. [6]

Figura 4. Mapa de procesos

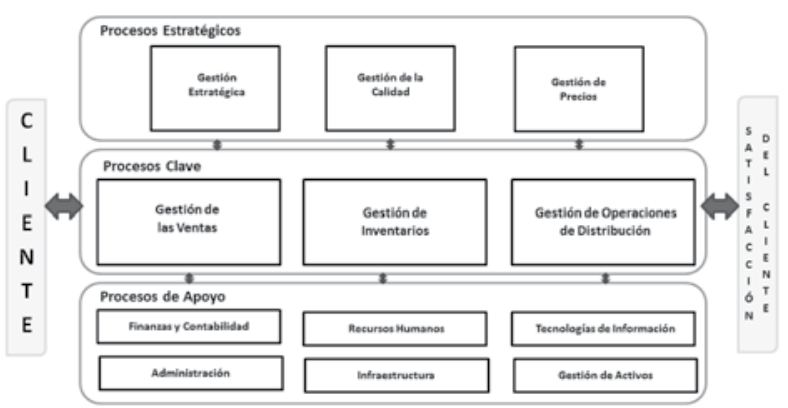

Fuente: Elaboración propia

La figura 4 nos muestra los tres tipos de procesos presentes en un mapeo. Primero, se observa los procesos estratégicos como Gestión Estratégica, de la Calidad y de Precios. Seguido, se aprecia los procesos clave como Gestión de las Ventas, de Inventarios y Operaciones de Distribución. Finalmente, los procesos de apoyo que están compuestos por Finanzas y Contabilidad, Administración, Recursos Humanos, Infraestructura, Tecnologías de Información y Gestión de Activos.

El proceso de Gestión de Operaciones de Distribución es el seleccionado como objeto de estudio y aplicación de BPM, por ser uno de los más importantes y transcendentales, ya que es núcleo y la razón de ser de este negocio. 


\subsection{DESCRIPCIÓN DEL SUBPROCESO DE PICKING}

El proceso clave de "Gestión de Operaciones de Distribución" se subdivide en cinco subprocesos:

- Recepción.- Se encarga de la recepción de los productos enviados por los proveedores.

- Almacenaje.- Se encarga de almacenar los productos recibidos en secciones de reserva que permitan una rápida reposición a las baldas de picking.

- Reposición.- Se encarga de colocar los productos de la sección de reserva a las baldas para se recogidas por los operarios de Picking.

- Picking.- Se encarga de la preparación de pedidos en base a las órdenes de trabajo.

- Distribución.- Se encarga de gestionar la distribución y entrega en forma oportuna de los pedidos a los locales solicitantes.

A continuación se procede a dar mayor detalle del subproceso de picking en el centro de distribución logístico:

\subsubsection{DEFINICIÓN}

Proceso mediante el cual los operadores del centro de distribución preparan los pedidos de los locales solicitantes en base a una orden de trabajo obtenida mediante el Sistema Automatizado de Radio Frecuencia. El Picking es efectuado en bandejas especiales que evitan el deterioro durante el traslado. Se utilizan bandejas de color rojo para el envío de productos que requieren un cuidado especial; los demás productos se recolectan en bandejas de color verde.

\subsubsection{PRINCIPALES OBJETIVOS DEL SUBPROCESO}

- Terminar la preparación de las órdenes de trabajo al $100 \%$, buscando cumplir una tasa de servicio alineada a los objetivos del área.

- Controlar y asegurar un adecuado llenado de las bandejas, tomando consideraciones como el tamaño del producto, su fragilidad y peso.

\subsubsection{FUNCIONES DEL OPERARIO}

- Efectuar todas las operaciones de preparación de pedidos en base a órdenes de trabajo.

- Asegurarse del perfecto estado y limpieza de las bandejas antes de empezar las labores.

- Disponer los productos al interior de las bandejas teniendo en cuenta el estado de los mismos, su fragilidad así como el embalaje adecuado para evitar deterioros durante el traslado a locales.

- Asegurarse que todos los embalajes estén debidamente etiquetados para su posterior entrega, con el fin de evitar errores y retrasos en el área de Tráfico.

- Ubicar las bandejas en las zonas de despacho manteniendo el orden según rutas de atención.

\subsection{CARACTERIZACIÓN DEL SUBPROCESO DE PICKING}

La caracterización de los procesos es parte fundamental de la correcta gestión de los mismos, permite adquirir una visión global de cada uno de los procesos debido a que se identifican sus factores y con ello la posibilidad de un control adecuado, la misma se muestra en la tabla 4.

Tabla 4. Caracterización del subproceso de picking

NOMBRE DEL PROCESO: Realizar picking

DUEÑO DEL PROCESO: Supervisor de picking

OBJETIVO: Terminar la preparación de las órdenes de trabajo al 100\%, buscando cumplir una tasa de servicio alineada a los objetivos del área

\begin{tabular}{|c|c|c|}
\hline \multirow{3}{*}{ 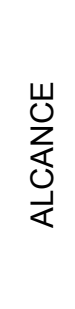 } & EMPIEZA & Generar reporte de pedidos por locales. \\
\hline & INCLUYE & $\begin{array}{c}\text { Generar tareas de preparación de pedidos, gestionar personal para los pedidos, } \\
\text { imprimir etiqueta para una bandeja de un pedido. }\end{array}$ \\
\hline & FINALIZA & Cerrar bandeja y colocar en zona de despacho. \\
\hline
\end{tabular}




\section{POLÍTICAS Y LINEAMIENTOS QUE APLICAN AL PROCESO}

Colores diferenciados en las bandejas acorde a las características del producto.

Manejo y trato diferenciado de acuerdo al tipo de producto.

\begin{tabular}{|l|c|c|}
\hline \multicolumn{2}{|c|}{ LIDER SUBPROCESOS } & ÁREA \\
\hline Preparar Pedido & Operario de picking & Almacén \\
\hline
\end{tabular}

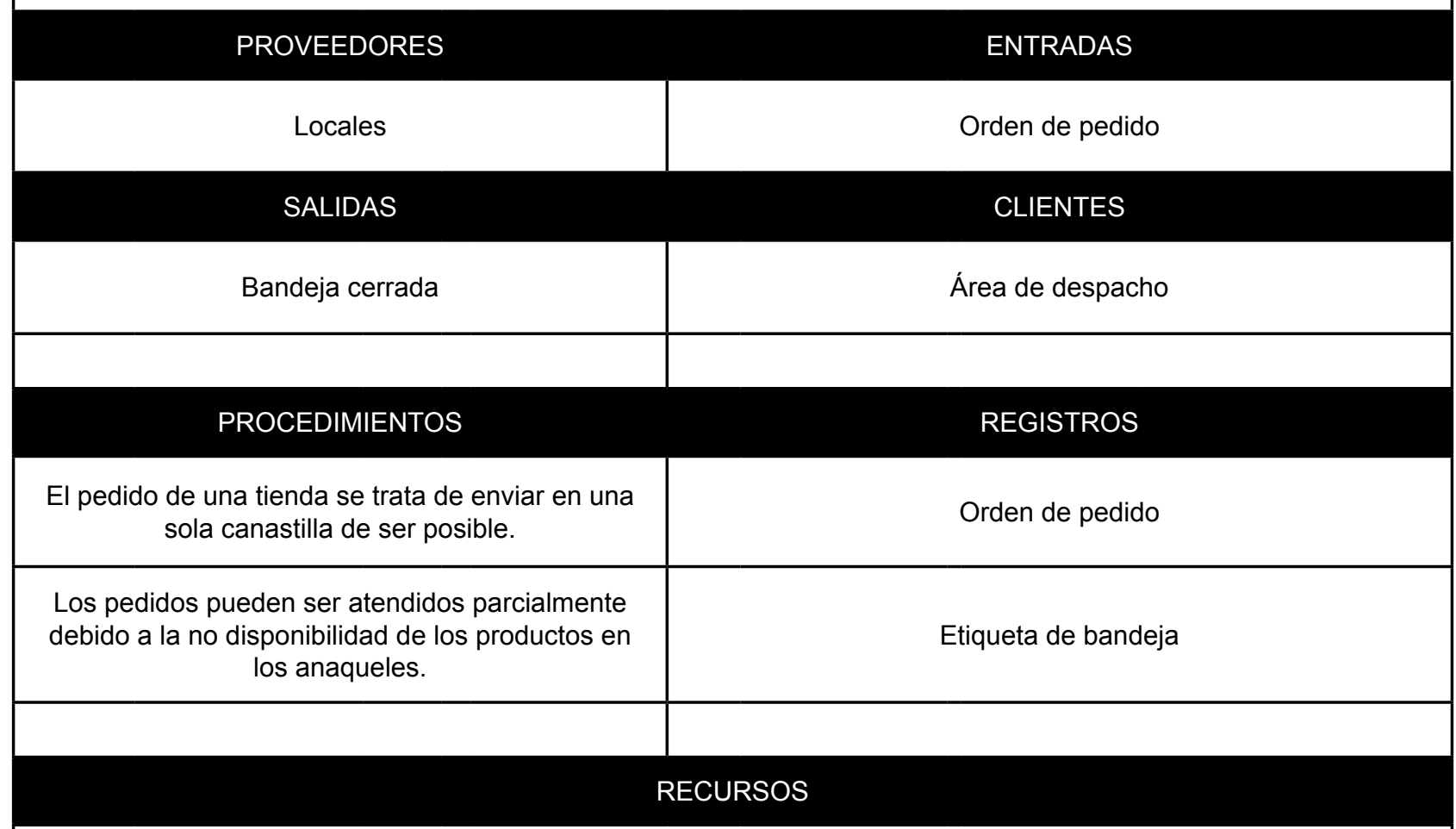

Sistema radio frecuencia, bandeja, coche, impresora, PC, etiquetas y personal

\begin{tabular}{|c|c|c|c|c|c|}
\hline INDICADORES & & FORMULA & \multicolumn{2}{|c|}{ PERIDIOCIDAD } & RESPONSABLE \\
\hline $\begin{array}{c}\text { Porcentaje de errores en } \\
\text { el proceso de picking de } \\
\text { la semana. }\end{array}$ & \multicolumn{2}{|c|}{$\frac{\text { Cant.Pedidos Errados }}{\text { Cant.Total Pedidos }} \times 100$} & \multicolumn{2}{|c|}{ Semanal } & Supervisor de picking \\
\hline \multicolumn{6}{|c|}{ VARIABLES DE CONTROL DEL PROCESO } \\
\hline \multicolumn{2}{|c|}{$\begin{array}{l}\text { Disponibilidad de productos en los } \\
\qquad \text { anaqueles }\end{array}$} & \multicolumn{2}{|c|}{ Cantidad de pedidos incompletos } & \multicolumn{2}{|c|}{ Cantidad de pedidos por atender. } \\
\hline
\end{tabular}

Fuente: Elaboración propia 


\subsection{AS-IS}

A continuación se presenta el modelado AS-IS del subproceso seleccionado.

Figura 5. Diagrama AS-IS del subproceso "Realizar Picking"

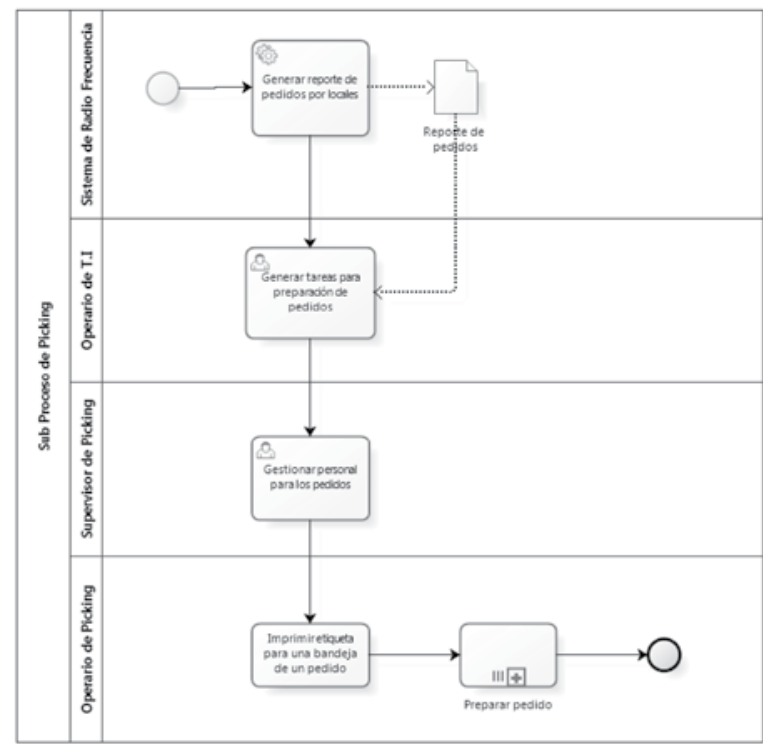

Fuente: Elaboración propia

Figura 6. Diagrama del subproceso "Preparar pedido"

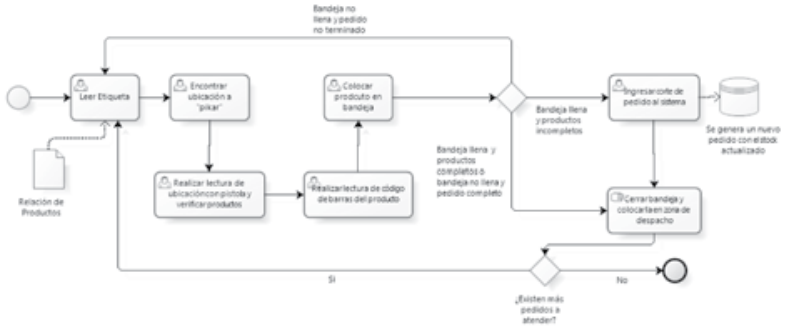

Fuente: Elaboración propia

EI AS-IS forma parte de las etapas de modelamiento y diseño del ciclo BPM. Muestra como se ejecuta un proceso actualmente, mediante el uso de flujos, conexiones, carriles y artefactos.

La figura 5 muestra el diagrama AS-IS del subproceso de picking. Empieza con la generación de los reportes de pedidos mediante un sistema de radio frecuencia automatizada. Luego, la información es recibida y procesada por un operador de tecnologías de información que se encarga de generar las tareas para la preparación.

El supervisor de picking recibe las tareas y procede a distribuirlas entre los operarios que tiene a su cargo. Enseguida cada operario procede a imprimir la etiqueta para la bandeja a llenar y procede con el subproceso de "Preparar Pedido" que en resumen se encarga de recolectar cada uno de los produc- tos de la orden de trabajo para finalmente, cerrar la bandeja y colocarla en la zona de despacho tal cual se aprecia en la figura 6 .

El principal problema del subproceso de picking son los errores que cometen los operarios al recolectar los productos y registrarlos como válidos en el sistema. Existe una verificación basada en muestras de este picking pero resulta ser insuficiente, ya que se reportan errores reiterativos por parte de los locales solicitantes.

Como solución de mejora se propone agregar un operario de calidad que se encargue de verificar los productos ingresados en las bandejas previo a su despacho. Debido a una limitación de tiempo y recursos, no se recomienda revisar todos los pedidos. En su lugar, se deben manejar estadísticas de los operarios de picking con mayor ratio de error en la recolección, la cual se actualizará diariamente. Con ello, el operario de calidad revisa únicamente los pedidos de un grupo reducido y ello no impacta en los tiempos de entrega.

\subsection{TO-BE}

A continuación se presenta el modelado TO-BE del subproceso seleccionado.

Figura 7: Diagrama TO-BE del subproceso "Realizar Picking"

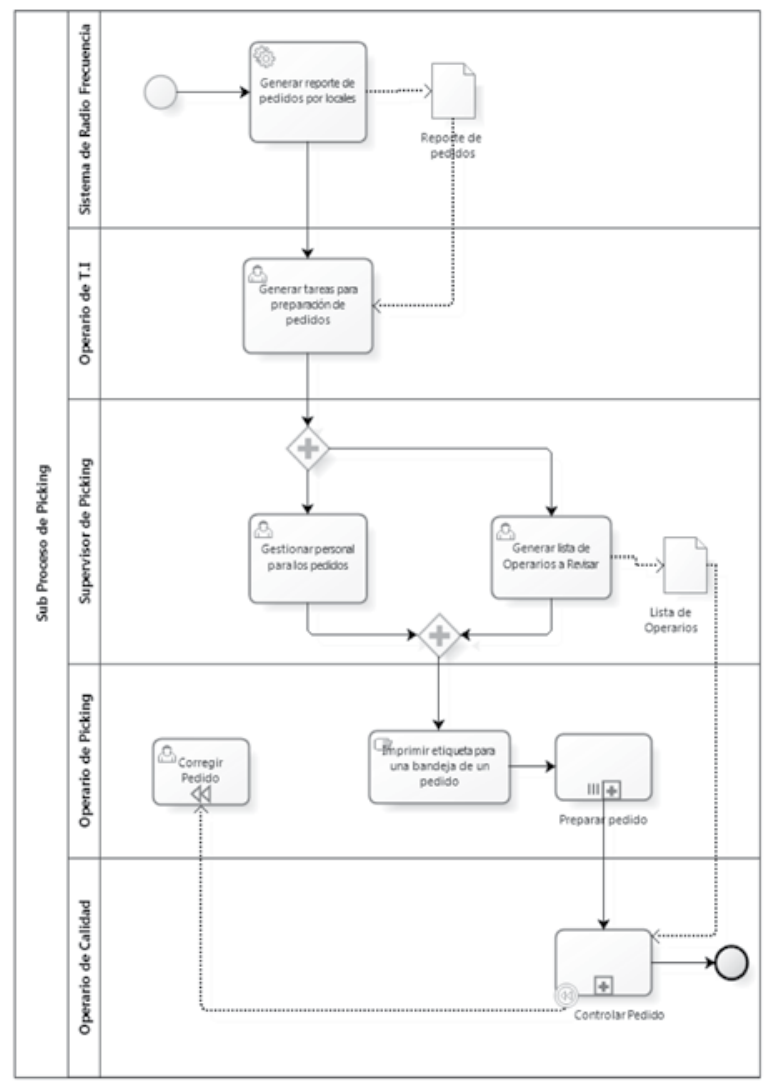

Fuente: Elaboración propia 
Figura 8. Diagrama del subproceso "Controlar pedido"

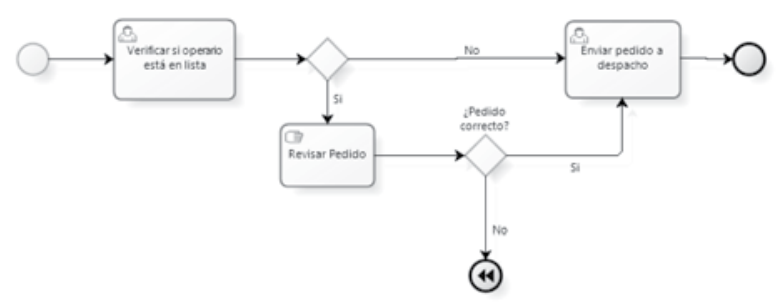

Fuente: Elaboración propia

El diagrama TO-BE de la figura 7 muestra la solución planteada para el problema de fallas en el subproceso de picking por parte de los operarios encargados de la recolección de productos.

Se aprecia que se utiliza una compuerta paralela para las actividades gestión de personal para los pedidos así como para la generación de la lista de operarios cuyas bandejas serán revisadas por el operario de calidad. Adicionalmente, se observa la agregación del subproceso "Controlar Pedido" cuyo detalle se muestra en la figura 8. Es aquí donde el operario de calidad procede a verificar las bandejas de los operarios de picking listados.

De presentarse alguna inconsistencia entre lo revisado y el pedido, inmediatamente se notifica al operario de picking para su corrección. Esto se aprecia en la figura 7 mediante el evento intermedio de compensación del subproceso "Controlar Pedido" que direcciona a la actividad "Corregir Pedido".

\section{CONCLUSIONES}

1. Se realizó una adecuada diagramación del mapa de procesos de la botica seleccionada. Se identificaron correctamente los procesos estratégicos, claves y operativos.

2. Se realizó una adecuada caracterización del subproceso de picking perteneciente al proceso clave de gestión de operaciones de distribu- ción. Ésta caracterización provee datos importantes para el entendimiento, alcance, medición y control del subproceso.

3. Por último, los diseños AS-IS y TO-BE se diagramaron correctamente, aplicando la notación BPMN y los diversos artefactos que lo componen. El diagrama TO-BE presenta la solución planteada de añadir el operario de calidad para la revisión de bandejas de los operarios de picking con mayor tasa de errores, que a futuro permitirá reducir la cantidad de errores en los pedidos.

\section{BIBLIOGRAFÍA}

[8] Bulsuk K. (2009), Taking the First Step with the PDCA (Plan-Do-Check-Act) Cycle. http://www. bulsuk.com/2009/02/taking-first-step-with-pdca.html\#axzz1xSJz6GUP (Visitado el 10-062012).

[8] Gartner. (2012). IT Glossary. http://www.gartner.com/it-glossary/business-process-management-bpm/ (Visitado el 12-06-2012).

[8] Jeston J. Nelis J. (2006). Business Process Management, Practical Guidelines to Successful Implementations. First Edition, Butterworth - Heinemann publications, UK.

[8] Object Management Group. (2011). Business Process Management (BPMN) Version 2.0, USA.

[8] Rabu T. (2011). Business Process Management life-cycle.http://businessprocess-managementnarotama. blogspot.com/2011/10/ bpm-life-cycle.html (Visitado el 01-06-2012).

[8] White S. Miers D. (2008). BPMN Modeling and Reference Guide: Understanding and Using BPMN. Digital Edition, Future Strategies Inc. USA. 